Supporting Information

\title{
Cerium Oxide Nanoparticles Stabilized within Metal-Organic Frameworks for the Degradation of Nerve Agents
}

Mohamed H. Hassan, Daniel Andreescu, and Silvana Andreescu*

Department of Chemistry and Biomolecular Science, Clarkson University, Potsdam, New York 13699-5810, USA.

* eandrees@clarkson.edu 


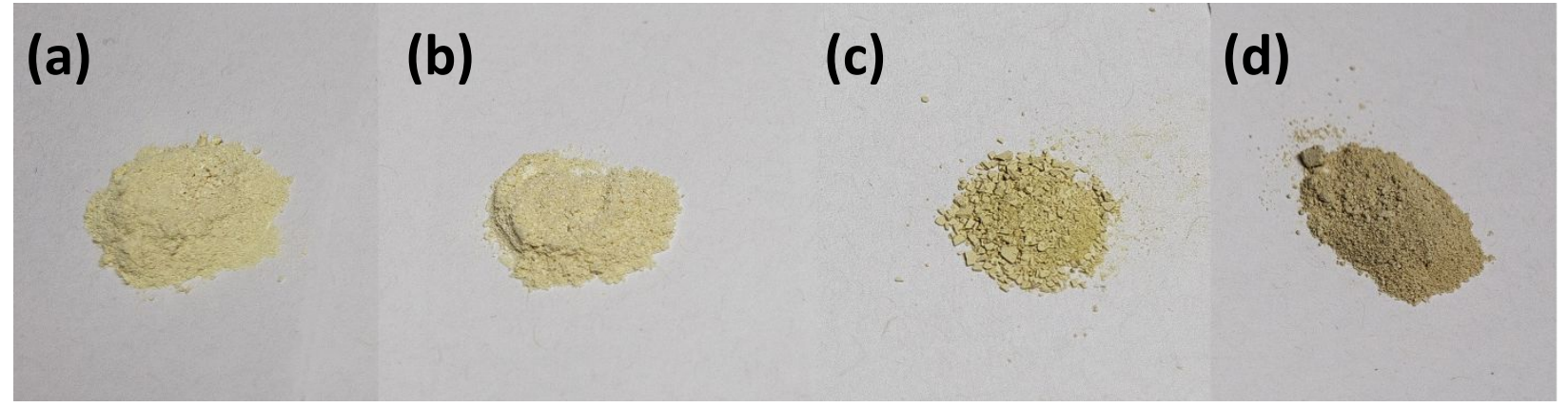

Figure S1. Optical images of (a) Ce-MOF, (b) CeNPs@MOF(I), (c) CeNPs@MOF(II), and (d) CeNPs@MOF(III) showing the color change after CeNPs loading.

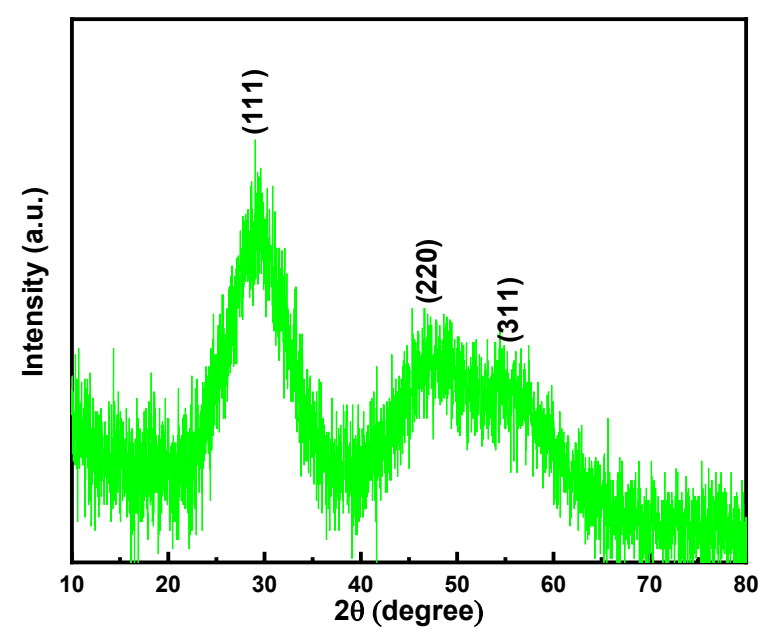

Figure S2. XRD pattern of CeNPs. 


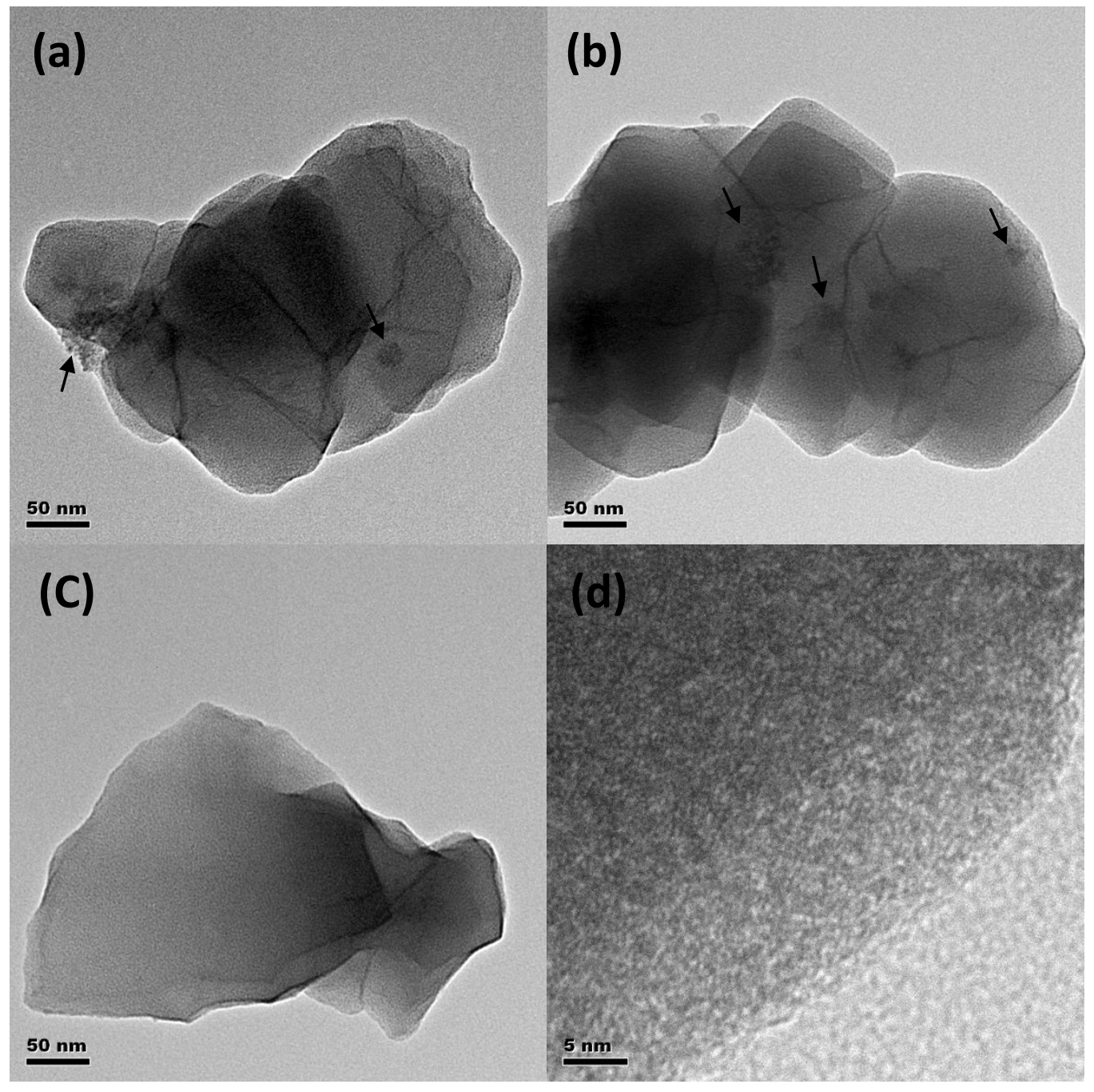

Figure S3. TEM images of (a,b) CeNPs@MOF(III), and (c,d) Ce-MOF. Arrows showing the presence of agglomerated CeNPs. 

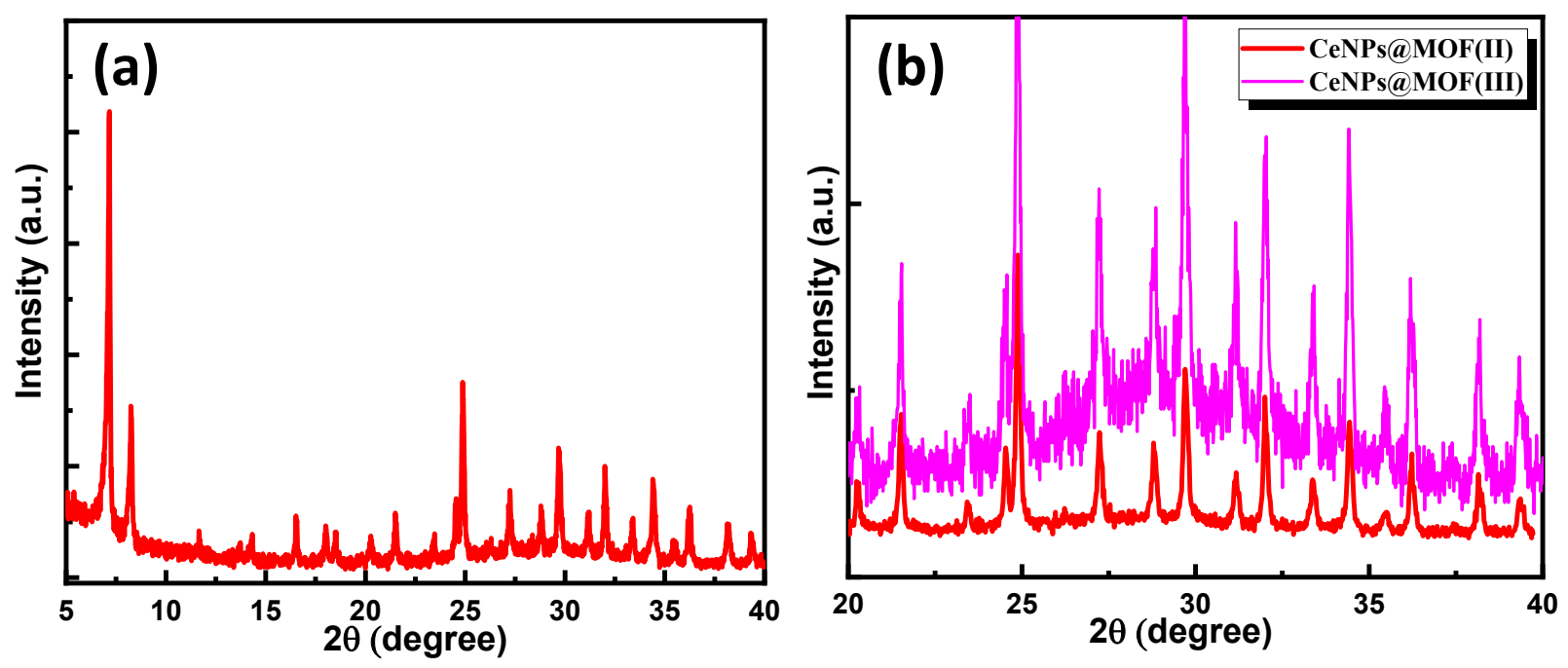

Figure S4. XRD patterns of (a) CeNPs@MOF(II) after DMNP degradation, and (b) CeNPs@MOF(II and III) (zoomed in view) showing the increased intensity of CeNPs (111) reflection in CeNPs@MOF(III) due to the higher CeNPs content. 